Original Article

\title{
Preoperative Increasing C-reactive Protein Affects the Outcome for Active Infective Endocarditis
}

Yuko Okada, MD, Mitsuharu Hosono, MD, Yasuyuki Sasaki, MD,

Hidekazu Hirai, MD, and Shigefumi Suehiro, MD

\begin{abstract}
Purpose: This study aimed to determine if preoperative time course changes in serum C-reactive protein (CRP) levels can predict clinical outcome of surgical intervention for active infective endocarditis.

Methods: Surgically treated patients $(n=109)$ with active infective endocarditis were reviewed retrospectively. We divided the patients into 2 subgroups according to preoperative transition of increasing or decreasing serum CRP levels, and performed a comparative study. The increasing CRP group included 29 patients and the decreasing CRP group included 80 patients.

Results: There were more patients with methicillin-resistant Staphylococcus aureus and New York Heart Association functional class IV in the increasing CRP group. Hospital mortality was significantly higher in the increasing CRP group (34.5\%) than that in the decreasing CRP group $(5.0 \%)(p<0.05)$. In multivariate analysis, 3 significant risk factors of surgical outcome were identified: a tendency for increasing preoperative CRP levels (odds ratio [OR]: 18.15, 95\% confidence interval [CI]: 1.03-320.78), nosocomial infective endocarditis (OR: 18.73, 95\% CI: 1.57-223.60), and dialysis (OR: 1025.46, 95\% CI: 2.89-363587.12).

Conclusion: The outcome of operations for patients with increasing preoperative CRP levels is poor. For treatment of active infective endocarditis, a better operative result is expected when preoperative CRP levels are decreasing.
\end{abstract}

Keywords: infective endocarditis, c-reactive protein, valve replacement

\section{Introduction}

Infective endocarditis (IE) is a serious infectious disease, and presents with a variety of symptoms and signs.

Department of Cardiovascular Surgery, Osaka City University Graduate School of Medicine, Osaka, Japan

Received: July 6, 2012; Accepted: August 21, 2012

Corresponding author: Yuko Okada, MD. Department of Cardiovascular Surgery, Osaka City University Graduate School of Medicine, 1-4-3 Asahi-machi, Abeno-ku, Osaka 545-8585, Japan Email: m2031129@med.osaka-cu.ac.jp

(C)2013 The Editorial Committee of Annals of Thoracic and Cardiovascular Surgery. All rights reserved.
Recently, early surgical treatment for IE has improved outcome, but some cases are difficult. Several factors are known to affect clinical outcome of active IE. Rostagno, et al. ${ }^{1)}$ reported that advanced age, severity of hemodynamic impairment and septic state were significantly related to clinical outcome in patients with IE. Other investigations have shown that risk factors of IE are Staphylococcus aureus infection, prosthetic valve endocarditis, vegetation length $>15 \mathrm{~mm}$, serum creatinine level $>2 \mathrm{mg}$ / $\mathrm{dl}$, abscess, and New York Heart Association (NYHA) functional class III to IV. ${ }^{1-3)}$ Among these factors, C-reactive protein (CRP) levels in IE have been the focus of several studies. Serum CRP levels have been used as a laboratory marker of inflammation applied to monitor the 
infection. ${ }^{4,5)}$ Some previous studies investigated the prognostic value of serial CRP measurements in patients with IE. ${ }^{6-8)}$ Olaison, et al. ${ }^{6}$ ) showed that elevated CRP levels were significantly prolonged in patients with complicated courses compared with simple courses.

Based on the findings from these studies, serum CRP levels may be correlated with the outcome of IE after clinical treatment. However, to the best of our knowledge, there are no previous reports on the correlation between preoperative serum CRP levels and operative results for active IE. The objective of this retrospective study was to determine if a preoperative time course change in serum CRP levels is a predictor of surgical outcome in patients with active IE.

\section{Materials and Methods}

\section{Patients}

One hundred thirteen consecutive patients underwent valve surgery for active IE at Osaka City University hospital (Osaka, Japan) from April 1990 to April 2010. Active IE was defined according to the Duke Endocarditis Service criteria. ${ }^{9}{ }^{10)}$ In 4 patients, CRP levels were measured only once until the operation. Since these 4 patients were excluded from this study, 109 patients were studied retrospectively. The patients comprised 72 males and 37 females, with a mean age of $54.9 \pm 14.6$ years. There were 24 patients $(22.0 \%)$ with prosthetic valve endocarditis. Nosocomial IE was observed in 17 patients.

\section{Perioperative management}

Antibiotics shown to be sensitive by blood culture test were used before and after surgery. In patients with negative results for blood cultures, penicillin and aminoglycoside were used empirically. The standard duration of antimicrobial treatment was defined according to the American Heart Association guidelines. ${ }^{9,10)}$

\section{Surgical indications}

The timing of operation mainly depended on the cardiologist's decision. Our surgical approach to the patient with active IE is as follows: (1) to operate before hemodynamic deterioration occurs if there is severe regurgitation; (2) to operate earlier if echocardiography reveals a vegetation (larger than $1.0 \mathrm{~cm}$ in diameter) that is considered to pose a high risk for embolism; and (3) to operate earlier if uncontrollable infection is considered to cause multiple organ failure or disseminated intravascular coagulopathy. Based on the results of a Japanese multicenter retrospective study ${ }^{11}$ in patients with cerebrovascular events, the established intervals between the onset of a cerebrovascular event and surgical intervention are at least 2 weeks in cerebral infarction cases and 4 weeks in hemorrhagic cases at our hospital. However, in cases with uncontrollable infection or progressive heart failure, we had to perform surgery within 2 weeks, even when there were cerebrovascular complications.

\section{CRP measurements}

CRP measurements were obtained in laboratory tests of the participating hospitals. CRP levels were measured at the first visit to our hospital, and routinely (every 2-3 days) during perioperative days. We divided the patients into 2 subgroups according to preoperative transition of serum CRP levels: the increasing CRP group $(n=29)$ and the decreasing CRP group $(n=80)$. In increasing CRP group patients, preoperative CRP levels were in the process of increasing since admission. In the decreasing group, CRP levels were in the process of decreasing since admission. We performed a comparative study between these 2 groups regarding perioperative data.

\section{Data collection and statistical analysis}

All clinical data were reviewed retrospectively. Clinical data were extracted from the medical records or by direct questioning or examination of patients. Individual consent for this study was performed at the same time as the explanation for undergoing cardiac surgery. Continuous variables are expressed as the mean \pm standard deviation. Direct variables are expressed as absolute numbers or percentages. The data were analyzed using StatView 5.0 (SAS Institute Inc., Cary, NC, USA). Comparative study between the 2 groups was performed by the Mann-Whitney U test for continuous variables, and the chi-square test and Fisher's exact probability test for categorical variables. Values of $\mathrm{p}<0.05$ were considered statistically significant.

We also conducted univariate and multivariate logistic regression analysis to determine independent risk factors of hospital mortality after surgical intervention for active IE. The significant factors in univariate analysis ( $p$ <.05) were forward analyzed with multivariate analysis to identify the independent risk factors.

\section{Results}

\section{Clinical characteristics}

The clinical characteristics of the increasing and decreasing CRP groups are summarized in Table 1. The 
Table 1 Patients' profiles and clinical characteristics

\begin{tabular}{lccc}
\hline Characteristics & $\begin{array}{c}\text { Increasing CRP group } \\
(\mathrm{n}=29)\end{array}$ & $\begin{array}{c}\text { Decreasing CRP group } \\
(\mathrm{n}=80)\end{array}$ & p value \\
\hline Age (years) & $60.5 \pm 14.1$ & $53.3 \pm 14.6$ & 0.008 \\
Sex (male/female) & $7: 22$ & $50: 30$ & 0.193 \\
Body surface area $\left(\mathrm{cm}^{2}\right)$ & $1.62 \pm 0.19$ & $1.55 \pm 0.17$ & 0.057 \\
Smoking & 13 & 33 & 0.738 \\
Hypertension & 10 & 15 & 0.084 \\
Diabetes mellitus & 8 & 14 & 0.246 \\
Hyperlipidemia & 6 & 11 & 0.378 \\
Low respiratory function & 10 & 12 & 0.246 \\
Renal disturbance $($ Cre $>1.5 \mathrm{mg} / \mathrm{dl})$ & 6 & 10 & 0.286 \\
Hemodialysis & 4 & 3 & 0.058 \\
Hepatopathy & 7 & 15 & 0.369 \\
History of cerebrovascular events & 10 & 22 & 0.479 \\
History of embolism & 12 & 30 & 0.713 \\
Nosocomial & 6 & 11 & 0.378 \\
MRSA infection & 6 & 3 & 0.005 \\
Arrhythmia & 2 & 12 & 0.256 \\
Left ventricular EF $(\%)$ & $57.2 \pm 11.1$ & $61.6 \pm 9.9$ & 0.111 \\
NYHA IV & 13 & 20 & 0.047 \\
CRP just before operation (mg/dl) & $11.5 \pm 9.2$ & $4.0 \pm 4.2$ & $<0.001$ \\
First measured serum CRP (mg/dl) & $7.1 \pm 7.3$ & $7.6 \pm 6.1$ & 0.598 \\
Interval until operation (days) & $7.3 \pm 11.8$ & $16.9 \pm 21.0$ & 0.012 \\
Prosthetic valve endocarditis & 6 & 18 & 0.840 \\
Preoperative identification of the causative & 14 & 46 & 0.392 \\
$\quad$ microorganism & & & \\
\hline Values are expressed & & & \\
\hline
\end{tabular}

Values are expressed as mean \pm SD or number. Cre: serum creatinine; CRP: C-reactive protein; EF: ejection fraction; MRSA: methicillin-resistant Staphylococcus aureus; NYHA: New York Heart Association functional class

mean age of increasing CRP group patients was significantly higher than that of the decreasing CRP group $(\mathrm{p}<0.01$. There was no significant difference in CRP levels at admission between the increasing and decreasing CRP groups, but serum CRP levels just before the operation were significantly higher in the increasing CRP group compared with those in the decreasing CRP group $(p<0.001)$. There were more patients on hemodialysis in the increasing CRP group compared with the decreasing CRP group, but this difference was not significant. The causative organisms were Staphylococcus in 45 patients, Streptococcus in 30 patients, gram-negative bacterium in 4 patients, fungus in 1 patient, others in 8 patients, and unknown in 21 patients (Table 2). Methicillinresistant Staphylococcus was observed in 16 patients. There were significantly more patients with IE caused by methicillin-resistant Staphylococcus aureus (MRSA) in the increasing CRP group than in the decreasing CRP group ( $\mathrm{p}<0.01)$. There were no significant differences in other causative organisms between the groups. Causative microorganisms were identified in 60 patients before the operation. However, even in 13 of 49 patients whose causative microorganism was unclear before surgery,
Table 2 Causative organisms of active IE

\begin{tabular}{lcc}
\hline Causative organism & $\begin{array}{c}\text { Increasing CRP } \\
\text { group }(\mathrm{n}=29)\end{array}$ & $\begin{array}{c}\text { Decreasing CRP } \\
\text { group }(\mathrm{n}=80)\end{array}$ \\
\hline Staphylococcus & 17 & 28 \\
Staphylococcus aureus & 7 & 12 \\
(MRSA ) & $(6)$ & $(3)$ \\
CNS & 10 & 16 \\
(MRCNS) & $(1)$ & $(6)$ \\
Streptococcus & 6 & 24 \\
Enterococcus & 0 & 6 \\
Gram-positive & 0 & 2 \\
Gram-negative & 1 & 3 \\
Fungus & 0 & 1 \\
Negative & 5 & 16 \\
\hline
\end{tabular}

CRP: C-reactive protein; CNS: Coagulase-negative Staphylococcus IE: infective endocarditis; MRCNS: methicillin resistant coagulase negative staphylococci; MRSA: methicillin-resistant Staphylococcus aureus

effective antibiotics were used before identification of these microorganisms. As mentioned above, the causative microorganism was unknown to the end of treatment in 21 patients. There was no significant difference between the 2 groups in the number of times there was preoperative identification of the causative microorganism. 
Table 3 Operative data

\begin{tabular}{lccc}
\hline Characteristics & $\begin{array}{c}\text { Increasing CRP } \\
\text { group }(\mathrm{n}=29)\end{array}$ & $\begin{array}{c}\text { Decreasing CRP } \\
\text { group (n=80) }\end{array}$ & p value \\
\hline Aortic valve surgery & 8 & 26 & 0.625 \\
Mitral valve surgery & 12 & 28 & 0.541 \\
Tricuspid valve surgery & 1 & 4 & 0.732 \\
Multiple valves & 7 & 18 & 0.857 \\
Valve replacement & 27 & 73 & 0.756 \\
Valvuloplasty & 1 & 4 & 0.732 \\
Prosthetic valve endocarditis & 6 & 18 & 0.840 \\
Annular abscess formation & 8 & 14 & 0.246 \\
Surgical indication & 8 & 27 & 0.543 \\
uncontrollable heart failure & 14 & 50 & 0.183 \\
uncontrollable infection & 12 & 34 & 0.917 \\
embolic events or fragile vegetation & $366.3 \pm 112.7$ & $330.6 \pm 137.4$ & 0.052 \\
Operation time (minutes) & $212.2 \pm 77.8$ & $173.2 \pm 85.5$ & 0.004 \\
ECC time (minutes) & 5 & 2 & 0.006 \\
IABP support & 3 & 0 & 0.004 \\
PCPS support & 4 & 6 & 0.315 \\
Aortic root reconstruction & 9 & 13 & 0.089 \\
Reoperation & & & \\
\hline Values are expressed as mean \pm SD or number. CRP: C-reactive protein; ECC: extra-corporeal \\
circulation; IABP: intra-aortic balloon pumping; PCPS: percutaneous cardio pulmonary \\
support & & & \\
& & &
\end{tabular}

Preoperative antibiotics included penicillin G in 19 patients, penicillin $\mathrm{G}$ plus aminoglycosides in 13 patients, vancomycin in 5 patients, carbapenems in 8 patients, cephalosporins in 9 patients, penicillin $\mathrm{G}$ plus cephalosporins in 6 patients, vancomycin plus aminoglycosides in 5 patients, vancomycin plus cephalosporins in 8 patients and others in 7 patients.

\section{Operative data}

The operative indications were uncontrollable heart failure, uncontrollable infection, and embolic events or fragile vegetation, but there were no significant differences in the operative indications between the 2 groups (Table 3). We performed isolated aortic valve surgery in 33 patients, isolated mitral valve surgery in 40 patients, isolated tricuspid valve surgery in 4 patients, multiple valve surgery in 25 patients, aortic root replacement in 3 patients, and other procedures in 4 patients. There were no significant differences in operative procedures and types of prosthetic valves between the groups. Although there was no significant difference in the operation time, the mean extracorporeal circulation time in the increasing CRP group was longer than that in the decreasing CRP group ( $\mathrm{p}<0.01)$. Postoperative intra-aortic balloon pumping or percutaneous cardiopulmonary support was required more frequently in the increasing CRP group than in the decreasing CRP group $(\mathrm{p}<0.01)$.

\section{Operative results}

Death in hospital occurred in 14 patients (12.8\%), and the causes of deaths included cardiac failure in 4 and cerebral infarction or cerebral bleeding in 3 patients. The causes of the other 7 deaths were multiple organ failure, hepatic failure, sepsis and pneumonia. Hospital mortality was significantly higher in the increasing CRP group (34.5\%) than that in the decreasing CRP group (5.0\%) $(\mathrm{p}<0.001)$. Intensive care unit stay in the increasing CRP group was significantly longer than that in the decreasing CRP group $(\mathrm{p}<0.01)$. Postoperative dialysis was required more often in the increasing CRP group $(\mathrm{p}<0.001)$, and postoperative low output syndrome was more frequent in the increasing CRP group $(\mathrm{p}<0.05)$ compared with the decreasing CRP group. There was no significant difference in the incidence of postoperative complications.

\section{Risk factor analysis}

In univariate analysis of the risk for in-hospital mortality, factors such as age, diabetes mellitus, a high level of CRP just before the operation, MRSA, lung damage, renal disturbance, dialysis, nosocomial IE, NYHA class IV, and increasing preoperative CRP levels were significant ( $<<0.05$, Table 4). When we conducted multivariate analysis, 3 significant risk factors were identified: increasing CRP levels (odds ratio [OR]: 18.15, $\mathrm{p}=0.048 ; 95 \%$ confidence interval $[\mathrm{CI}]:$ 1.03-320.78), 
Table 4 Risk factor analysis for operative mortality: univariate analysis

\begin{tabular}{|c|c|c|c|}
\hline Characteristics & Odds ratio & $95 \% \mathrm{CI}$ & $\mathrm{p}$ value \\
\hline Age & 1.10 & $1.03-1.16$ & 0.002 \\
\hline Sex (male/female) & 0.68 & $0.22-2.12$ & 0.501 \\
\hline Body surface area $\left(\mathrm{cm}^{2}\right)$ & 0.57 & $0.02-13.81$ & 0.609 \\
\hline Smoking & 0.73 & $0.23-2.35$ & 0.599 \\
\hline Hypertension & 1.41 & $0.40-4.95$ & 0.592 \\
\hline Diabetes mellitus & 5.33 & $1.63-17.43$ & 0.003 \\
\hline Hyperlipidemia & 0.89 & $0.18-4.38$ & 0.885 \\
\hline Low respiratory function & 7.71 & $2.32-25.64$ & $<0.001$ \\
\hline Renal disturbance $(\mathrm{Cre}>1.5 \mathrm{mg} / \mathrm{dl})$ & 4.24 & $1.20-14.97$ & 0.025 \\
\hline Hemodialysis & 70.5 & $7.53-660.18$ & $<0.001$ \\
\hline Hepatopathy & 1.84 & $0.51-6.55$ & 0.355 \\
\hline History of cerebrovascular events & 1.33 & $0.41-4.32$ & 0.635 \\
\hline History of embolism & 1.23 & $0.39-3.83$ & 0.722 \\
\hline Nosocomial & 12.74 & $3.61-45.00$ & $<0.001$ \\
\hline MRSA & 23.00 & $4.82-109.79$ & $<0.001$ \\
\hline Prosthetic valve endocarditis & 0.91 & $0.25-3.77$ & 0.954 \\
\hline Arrhythmia & 5.31 & $1.46-19.31$ & 0.487 \\
\hline Left ventricular EF (\%) & 1.00 & $0.94-1.06$ & 0.884 \\
\hline NYHA IV & 0.32 & $2.24-27.26$ & $<0.001$ \\
\hline Preoperative serum CRP (mg/dl) & 1.09 & $1.01-1.18$ & 0.021 \\
\hline Increasing preoperative CRP $(\mathrm{mg} / \mathrm{dl})$ & 10.00 & $2.83-35.39$ & $<0.001$ \\
\hline Interval until operation (days) & 1.00 & $0.98-1.03$ & 0.670 \\
\hline $\begin{array}{l}\text { Preoperative identification of the causative } \\
\text { microorganism }\end{array}$ & 1.55 & $0.48-4.98$ & 0.459 \\
\hline
\end{tabular}

nosocomial IE (OR: 18.73, $\mathrm{p}=0.021 ; 95 \% \mathrm{CI}: 1.57-$ 223.60) and dialysis (OR: 1025.46, $\mathrm{p}=0.021 ; 95 \% \mathrm{CI}$ : 2.89-363587.12) (Table 5).

\section{Discussion}

This study clearly showed that preoperative increasing serum CRP levels, even with antimicrobial treatment, were associated with poor surgical results. To the best of our knowledge, there are no previous reports regarding the correlation between time course changes in preoperative serum CRP levels and surgical results of active IE. Although some other studies have focused on the value of CRP in IE, most of these studies involved a relatively small number of patients and limited or no statistics. Moreover, in some of them, only CRP values on admission were examined. ${ }^{7)}$ Verhagen, et al. reported that high CRP levels after 1 week of antimicrobial therapy and a slow percentage decline in CRP levels during the first week of treatment were indicators of poor clinical outcome in treatment of left-sided active IE. ${ }^{8)}$ Our present findings support their results, although their study did not present surgical results.
There are many indices of inflammation, such as total leukocyte count, CRP, interleukin-6, and procalcitonin. CRP is synthesized by the liver, mainly in response to interleukin-6 during infection. ${ }^{12-14)}$ Interleukin-6 levels decrease faster than CRP levels during antimicrobial treatment and they are useful for monitoring of treatment. ${ }^{13)}$ However, CRP is easier to measure in a blood sample than interleukin-6 in a general hospital. Jereb, et al. ${ }^{15}$ ) compared the accuracy of procalcitonin and CRP in diagnosing IE, but they failed to demonstrate superiority of procalcitonin as a diagnostic laboratorial parameter in predicting IE. ${ }^{12,15)}$ Based on these facts, we believe that a change in the time course of serum CRP levels is more suitable to guide therapeutic decisions in active IE than other indices.

The clinical usefulness of serial CRP measurements in monitoring the response to therapy in patients with septicemia has been well documented. Heiro, et al.7) showed that serial determinations of CRP values proved useful in monitoring of a patient's response to therapy of IE. Although a single value of serum CRP level is seldom useful to guide therapeutic decisions, multiple measures over time are useful. Previous results confirm 
Table 5 Risk factor analysis for operative mortality: multivariate analysis

\begin{tabular}{lccc}
\hline Characteristics & Odds ratio & $95 \%$ CI & p value \\
\hline Age & 1.06 & $0.96-1.17$ & 0.228 \\
Diabetes mellitus & 0.64 & $0.04-10.99$ & 0.755 \\
Low respiratory function & 2.05 & $0.15-28.73$ & 0.595 \\
Renal disturbance $($ Cre $>1.5 \mathrm{mg} / \mathrm{dl})$ & 0.32 & $0.02-6.13$ & 0.448 \\
Hemodialysis & 1025.46 & $2.89-363587.12$ & 0.021 \\
Nosocomial & 18.73 & $1.57-223.60$ & 0.021 \\
MRSA & 1.08 & $0.04-28.88$ & 0.962 \\
NYHA IV & 8.14 & $0.70-94.28$ & 0.093 \\
Preoperative serum CRP $(\mathrm{mg} / \mathrm{dl})$ & 1.07 & $0.87-1.32$ & 0.508 \\
Increasing preoperative CRP $(\mathrm{mg} / \mathrm{dl})$ & 18.15 & $1.03-320.78$ & 0.048 \\
\hline
\end{tabular}

CI: confidence interval; Cre: serum creatinine; CRP: C-reactive protein; MRSA: methicillin-resistant Staphylococcus aureus; NYHA: New York Heart Association functional class

that a progressive decline in serum CRP levels reflects a favorable response to antimicrobial therapy. ${ }^{16,17)}$ In the current study, preoperative identification of the causative microorganism did not influence the surgical outcomes. However, as a result, effective antibiotics were able to be used in some patients whose causative microorganism was unknown. From these facts, use and selection of effective antibiotics in the early stage of treatment for active IE appear to be more important than the identification of the causative microorganism. Additionally, the effectiveness of antibiotics can be determined by serum CRP levels.

In the current study, the increasing CRP group comprised many elderly patients, such as those with MRSA infections, and hemodialysis patients. In such patients, it may be difficult to achieve a favorable response to antimicrobial therapy for active IE. This could be the reason why the operative results in the increasing CRP group were poor.

Since surgical technical success does not always result in clinical success in treatment of active IE, clarifying the risk factors of surgical treatment for active IE is important. Previous studies have reported that advanced age, Staphylococcus aureus, prosthetic valve endocarditis, vegetation length, renal function impairment, abscess formation, severity of hemodynamic impairment and septic state are significantly related to clinical outcome in patients with IE. These previously reported risk factors were not only for surgical treatment but also for a variety of other treatments. In the present study, preoperative increasing CRP levels, as well as nosocomial IE and hemodialysis, were identified as risk factors of surgical treatment for active IE in multivariate analysis. In a previous report, ${ }^{18)}$ we demonstrated a poor surgical outcome of nosocomial IE. In such high risk conditions, we believe that an early decision and optimal timing of operation before deterioration of a patient's general condition are important to improve surgical outcome. However, the optimal timing of surgery is also difficult to determine because of a lack of evidence-based data. Heart failure has the greatest effect on prognosis, and moderate to severe heart failure results in surgery being performed in an urgent setting. Moreover, the size of vegetation is a predictor of embolism and death. ${ }^{19,20)}$ In such situations, early surgeries are indicated to avoid death and severe complications. Our study confirmed that increasing preoperative CRP levels are an indicator of poor surgical outcome. Therefore, we consider that an early operation should be performed for patients who are at risk, but if possible, it should be performed after a response to antibiotic therapy.

There are limitations to this study. The proportion of patients with NYHA functional class IV was higher and the interval between the initiation of medical therapy and the operation was shorter in the increasing CRP group than those in the decreasing CRP group. Heart failure can have a considerable effect on the operative outcome and prognosis in active IE. Our findings suggest that more urgent and uncontrollable patients were included in the increasing CRP group. Another possible reason is that patients with severe heart failure might have a poor response to antibiotic treatment and then require an urgent operation. Although there were no differences between the groups in the number of diseased valves, the frequency of prosthetic valve endocarditis and the frequency of annular abscess, more patients required postoperative intra-aortic balloon pumping or percutaneous cardiopulmonary support in the increasing CRP group compared with those in the decreasing CRP group. The reason for more postoperative low output syndrome observed in the increasing CRP group 
might also be correlated with preoperative NYHA functional class. Furthermore, since weaning from cardiopulmonary bypass was more difficult in patients of the increasing CRP group, the extracorporeal circulation time might become longer in this group. These issues might have affected the results of the present study, but NYHA class was not detected as an independent risk factor of surgical treatment in our study. Because of these study limitations, we suggest that CRP levels alone should not be used as a prognostic factor, but should be considered along with other clinical variables.

\section{Conclusion}

In conclusion, the outcome of operations for patients in whom CRP levels are increasing before the operation is poor. In treatment of active IE, a better operative result can be expected when preoperative CRP levels are decreasing.

\section{Disclosure Statement}

We have no conflict of interest.

\section{References}

1) Rostagno C, Rosso G, Puggelli F, et al. Active infective endocarditis: Clinical characteristics and factors related to hospital mortality. Cardiol J 2010; 17: 566-73.

2) Yasuhiro K, Toshifumi M, Hideyuki K, Norihiko S, Keishu Y, et al. Surgical treatment of active infective endocarditis: determinants of early outcome. The Japanese Society for Cardiovascular Surgery 2004; 33: $1-5$.

3) Rasmussen RV, Høst U, Arpi M, et al. Prevalence of infective endocarditis in patients with Staphylococcus aureus bacteraemia: the value of screening with echocardiography. Eur J Echocardiogr 2011; 12: 414-20.

4) Gabay C, Kushner I. Acute-phase proteins and other systemic responses to inflammation. N Engl J Med 1999; 340: 448-54.

5) Vigushin DM, Pepys MB, Hawkins PN. Metabolic and scintigraphic studies of radioiodinated human C-reactive protein in health and disease. J Clin Invest 1993; 91: 1351-7.

6) Olaison L, Hogevik H, Alestig K. Fever, C-reactive protein, and other acute-phase reactants during treatment of infective endocarditis. Arch Intern Med 1997; 157: 885-92.

7) Heiro M, Helenius H, Sundell J, et al. Utility of serum C-reactive protein in assessing the outcome of infective endocarditis. Eur Heart J 2005; 26: 1873-81.

8) Verhagen DW, Hermanides J, Korevaar JC, et al. Prognostic value of serial C-reactive protein measurements in left-sided native valve endocarditis. Arch Intern Med 2008; 168: 302-7.

9) Nishimura RA, Carabello BA, Faxon DP, et al. ACC/ AHA 2008 guideline update on valvular heart disease: focused update on infective endocarditis: a report of the American College of Cardiology/American Heart Association Task Force on Practice Guidelines: endorsed by the Society of Cardiovascular Anesthesiologists, Society for Cardiovascular Angiography and Interventions, and Society of Thoracic Surgeons. Circulation 2008; 118: 887-96.

10) Baddour LM, Wilson WR, Bayer AS, et al. Infective endocarditis: diagnosis, antimicrobial therapy, and management of complications: a statement for healthcare professionals from the Committee on Rheumatic Fever, Endocarditis, and Kawasaki Disease, Council on Cardiovascular Disease in the Young, and the Councils on Clinical Cardiology, Stroke, and Cardiovascular Surgery and Anesthesia, American Heart Association: endorsed by the Infectious Diseases Society of America. Circulation 2005; 111: e394-434.

11) Eishi K, Kawazoe K, Kuriyama Y, et al. Surgical management of infective endocarditis associated with cerebral complications. Multi-center retrospective study in Japan. J Thorac Cardiovasc Surg 1995; 110: 1745-55.

12) Ridker PM. Clinical application of C-reactive protein for cardiovascular disease detection and prevention. Circulation 2003; 107: 363-9.

13) Hryniewiecki T, Rawczynska-Englert I, Sitkiewicz D, et al. [Comparison of interleukin-6 and C-reactive protein serum concentrations assessment in diagnosis of infective endocarditis]. Pol Arch Med Wewn 2002; 108: $947-52$.

14) Simon L, Gauvin F, Amre DK, et al. Serum procalcitonin and C-reactive protein levels as markers of bacterial infection: a systematic review and meta-analysis. Clin Infect Dis 2004; 39: 206-17.

15) Jereb M, Kotar T, Jurca T, et al. Usefulness of procalcitonin for diagnosis of infective endocarditis. Intern Emerg Med 2009; 4: 221-6.

16) Roberts-Thomson PJ, Koh LY, Kennedy A, et al. Serological investigations in the diagnosis and management of infective endocarditis. Aust N Z J Med 1986; 16: 761-5.

17) McCartney AC, Orange GV, Pringle SD, et al. Serum C reactive protein in infective endocarditis. J Clin Pathol 1988; 41: 44-8.

18) Shibata T, Sasaki Y, Hirai H, et al. Early surgery for hospital-acquired and community-acquired active infective endocarditis. Interact Cardiovasc Thorac Surg 2007; 6: 354-7.

19) Thuny F, Beurtheret S, Mancini J, et al. The timing of surgery influences mortality and morbidity in adults with severe complicated infective endocarditis: a propensity analysis. Eur Heart J 2011; 32: 2027-33.

20) Thuny F, Habib G. When should we operate on patients with acute infective endocarditis? Heart 2010; 96: 892-7. 